\title{
Ramp lesions are frequently missed in ACL-deficient knees and should be repaired in case of instability
}

\author{
Alexander Bumberger ${ }^{1}$. Ulrich Koller ${ }^{1} \cdot$ Marcus Hofbauer $^{1} \cdot$ Thomas Manfred Tiefenboeck $^{1} \cdot$ Stefan Hajdu ${ }^{1}$. \\ Reinhard Windhager ${ }^{1} \cdot$ Wenzel Waldstein ${ }^{1}[0$
}

Received: 21 December 2018 / Accepted: 24 April 2019 / Published online: 10 May 2019

(c) The Author(s) 2019

\begin{abstract}
Purpose The aim of the current study was (1) to provide an overview of common definitions and classification systems of ramp lesions (RL) and (2) to systematically review the available literature with regard to the diagnosis and treatment of RLs in anterior cruciate ligament (ACL)-deficient knees.

Methods Following the PRISMA guidelines, MEDLINE and Scopus were searched for articles (1) reporting on acute or chronic ACL injuries, (2) with concomitant medial meniscus injury, (3) located at the posterior meniscocapsular attachment site (and red-red zone). Ex vivo studies, reviews and technical notes were excluded.

Results Twenty-seven studies were included based on the criteria mentioned above. RLs are common in ACL-deficient knees with a prevalence ranging from 9 to $24 \%$. RLs should especially be suspected in younger patients, patients with an increased meniscal slope and in patients with prolonged time from injury to surgery. The sensitivity of MRI for the detection of RLs ranges from 48 to $86 \%$ at a specificity of 79-99\%. For arthroscopy, RLs are easily missed through standard anterior portals (sensitivity 0-38\%). RL repair leads to a significant improvement of subjective knee scores, regardless of the specific fixation technique. For stable RLs, the literature suggests equivalent postoperative stability for trephination and abrasion compared to surgical RL repair.

Conclusion Ramp lesions are frequently missed in ACL-deficient knees on standard arthroscopy with anterior portals only. If a RL is suspected, exploration via an additional posteromedial portal is indicated. In case of instability, RL repair should be performed.
\end{abstract}

Level of evidence IV.

Keywords Ramp lesion · Meniscocapsular separation · Meniscocapsular attachment tear · ACL deficiency $\cdot$ Knee instability $\cdot$ Review

\section{Abbreviations}

ACL Anterior cruciate ligament

ACLR Anterior cruciate ligament reconstruction

ALLR Anterolateral ligament repair

HL Hidden lesion

IKDC International Knee Documentation Committee

LNS Lysholm Knee Score

TFI Time from injury

RL Ramp lesion

Wenzel Waldstein

wwaldstein@gmail.com

1 Department of Orthopaedics and Trauma Surgery, Vienna General Hospital, Medical University of Vienna, Waehringer Guertel 18-20, 1090 Vienna, Austria
MCS Menisco-capsular separation

PHMM Posterior horn of the medial meniscus

\section{Introduction}

The mechanisms of anterior cruciate ligament (ACL) rupture and concomitant knee injuries have been extensively investigated over the last decades, revealing that the minority of ACL ruptures occurs as an isolated injury $[12,20]$. Meniscal tears have been reported to be present in 55\% up to nearly $80 \%$ of ACL injuries, with significantly higher rates in chronically ACL-deficient knees [12, 26, 46]. Accordingly, time to surgery correlates with the incidence of concomitant meniscus injuries [25, 29]. In particular, the incidence of medial meniscus (MM) tears seems to significantly 
increase with delayed surgery $[26,50]$. Conversely, patients with medial or lateral meniscus instabilities have a significantly increased risk of ACL failure after reconstruction [32]. Hence, early ACL reconstruction (ACLR) and meniscal repair are recommended to prevent secondary meniscus injuries and improve the long-term outcome [12, 27].

Over the last years, more attention has been paid to tears located at the posteromedial meniscocapsular junction. Even though first characterizations date back at least 35 years [13], these injuries frequently remained unnoticed in knees with ACL injuries. Since their first description, a range of terms has been used synonymously to describe these lesions, including meniscocapsular separation (MCS), meniscosynovial tear, hidden lesion (HL) and ramp lesion (RL). The term ramp lesion was first used by Strobel in 1988, defining it as "a special type of meniscal injury involving the peripheral attachment of the posterior horn of the medial meniscus, typically associated with an ACL deficiency". Currently, there is no consensus regarding the definition of RLs [4] which has resulted in misleading descriptions. Some authors have also considered posteromedial meniscus tears in the red-red zone as ramp lesions [23, 43].

Thaunat et al. classified five lesion subtypes by their exact tear pattern, location, degree of mobility and visibility during arthroscopy [43]. Type 1 is meniscocapsular junction tears located in the synovial sheath with very low mobility at probing. Type 2 includes partial superior meniscus tears which are stable and can only be diagnosed via the trans-notch approach. Type 3 is partial inferior meniscus tears (hidden lesions) associated with meniscotibial ligament disruptions resulting in high probing mobility. Type 4 involves complete longitudinal vertical meniscus tears in the red-red zone. Type 5 describes double longitudinal vertical tears (Fig. 1).
Fig. 1 Illustration of five ramp lesion subtypes depending on tear pattern, location, degree of mobility and visibility during arthroscopy according to Thaunat et al. [43]
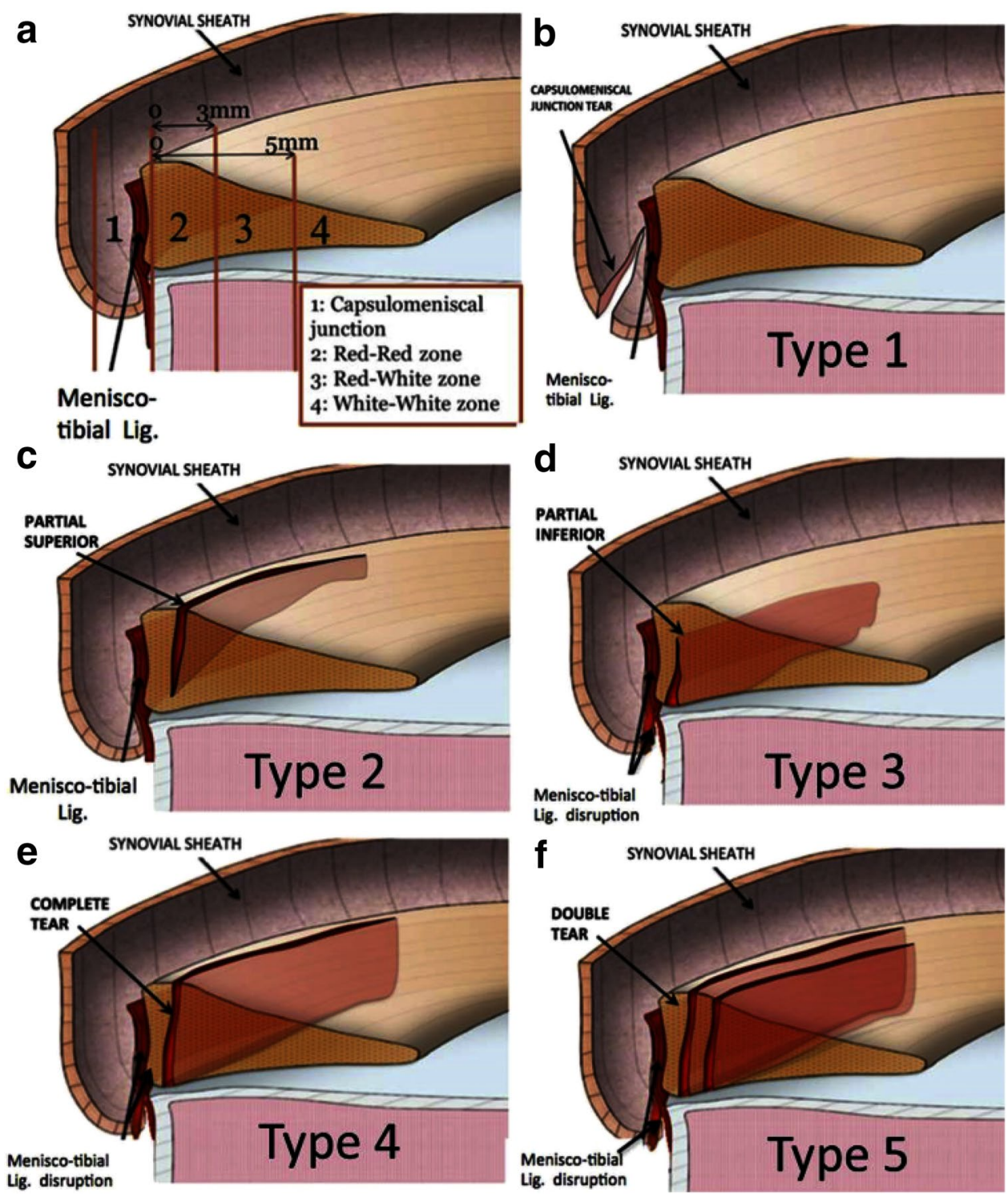
Lig. disruption

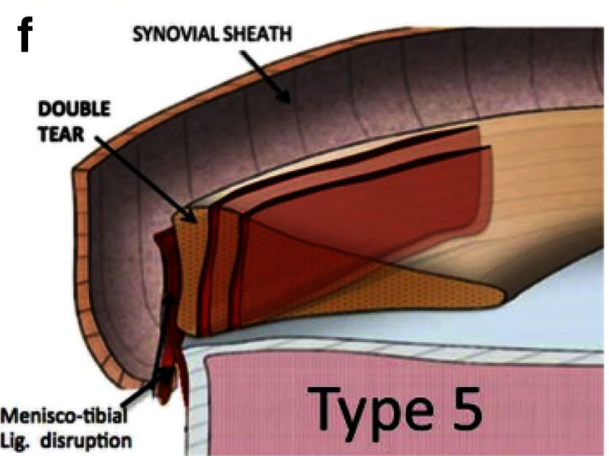


An alternative classification has been proposed by Seil et al., paying more attention to the mediolateral extent of lesions and the properties of the capsule-ligament complex depending on the degree of flexion [34]. Furthermore, there seems to be confusion concerning the term "peripheral" in the context of RLs, since it has been referred both to the meniscocapsular and meniscotibial attachment sites of the PHMM [5, 9], as well as to lesions in the RR zone [23, 44]. Table 1 provides an overview of different RL definitions.

This review was conducted due to the growing number of publications regarding posteromedial meniscocapsular attachment tears, as well as the inconsistent nomenclature. The aim of this study was (1) to provide an overview of common ramp lesion definitions and classification systems and (2) to systematically review the available literature with regard to the diagnosis and treatment of ramp lesions (Fig. 2).

\section{Materials and methods}

A systematic database search of the literature concerning ramp lesions in ACL-deficient knees utilising pre-defined search terms was performed (see addendum). The study was conducted according to the preferred reporting items for systematic reviews and meta-analysis (PRISMA) guidelines [28]. The methodological index for non-randomised studies (MINORS) was used to assess the quality of all included non-randomised studies. Included databases were Medline

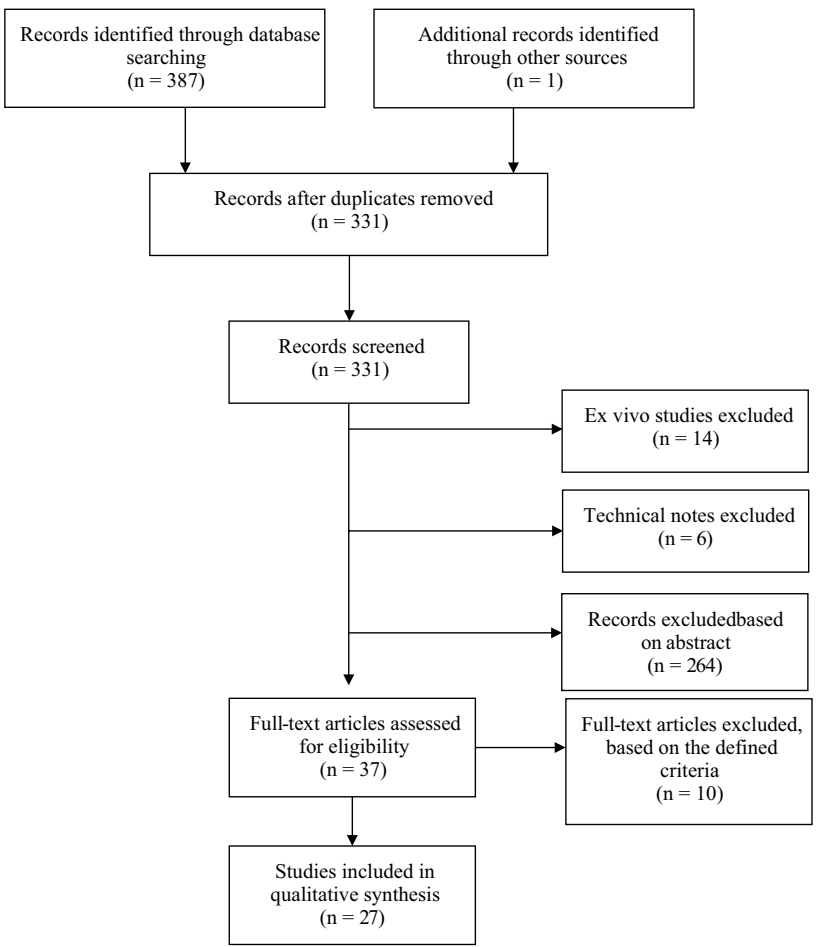

Fig. 2 Flow chart of study protocol following the PRISMA guidelines. The systematic review included 27 studies

and Scopus. English and German articles were considered because the authors speak both languages.

Table 1 Definitions of ramp lesions as described in the literature

\begin{tabular}{|c|c|c|}
\hline Authors & Year & RL definition \\
\hline \multicolumn{3}{|c|}{ Definitions not including RR zone tears } \\
\hline Di Vico et al. [9] & 2017 & $\begin{array}{l}\text { Ramp lesions and hidden lesions represent subgroups of longitudinal lesions involving the } \\
\text { peripheral attachment of the PHMM }\end{array}$ \\
\hline Seil et al. [34] & 2017 & Traumatic disruptions between the PHMM and its mensicoligamental junction \\
\hline Chen et al. [5] & 2017 & Peripheral attachment lesion of the PHMM \\
\hline Keyhani et al. [16] & 2016 & Longitudinal tear or detachment of the peripheral rim around the PHMM \\
\hline Li et al. [22] & 2015 & Peripheral attachment lesion of the PHMM \\
\hline Strobel et al. [42] & 1988 & $\begin{array}{l}\text { Meniscal injury involving the peripheral attachment of the PHMM and is typically associated } \\
\text { with an ACL deficiency }\end{array}$ \\
\hline \multicolumn{3}{|c|}{ Definitions including RR zone tears } \\
\hline Sonnery-Cottet et al. [40] & 2018 & $\begin{array}{l}\text { Disruption or tear of the peripheral meniscocapsular attachments of the posterior horn of the } \\
\text { medial meniscus (exclusion criteria suggests that RR zone is included) }\end{array}$ \\
\hline Kim et al. [18] & 2018 & Peripheral longitudinal tear within $4 \mathrm{~mm}$ of the meniscocapsular junction of PHMM \\
\hline Thaunat et al. $[43,44]$ & 2016 & Meniscosynovial or meniscocapsular tears + RR zone (see Fig. 1) \\
\hline Furumatsu et al. [11] & 2013 & $\begin{array}{l}\text { Peripheral longitudinal tears of the MM included partial- or full-thickness, simple longitudinal } \\
\text { tears } \geq 1 \mathrm{~cm} \text { in length located in the outer one-third of the posterior segment }\end{array}$ \\
\hline Liu et al. $[23,24]$ & 2011 & $\begin{array}{l}\text { Tear of the peripheral attachment of the posterior horn of the medial meniscus (synovial- } \\
\text { meniscus junction or red-red zone) }\end{array}$ \\
\hline Resnick et al. [31] & 2007 & Double vertical longitudinal tears of the PHMM \\
\hline
\end{tabular}

Some authors consider also lesions in the red-red zone as ramp lesion 
The search terms (see addendum) were assembled to cover a broad spectrum of meniscus and associated knee injuries, in order not to miss any relevant literature. As represented in our search terms, we paid special attention to keywords such as "ramp", "hidden", "meniscocapsular", "meniscosynovial" and "posteromedial" in the context of meniscus lesions. Next, relevant articles were preselected based on the abstract by two independent reviewers (AB and WW). A third reviewer (TT) was consulted to obtain consensus in case of discrepancy. Subsequently, the full text was carefully reviewed to check whether included lesions were (1) associated with acute or chronic ACL injury, (2) concerning the medial meniscus, and (3) located at the posterior meniscocapsular attachment site (and the periphery of the RR zone). Ex vivo studies, reviews and technical notes were excluded. Data regarding prevalence, diagnosis, surgical technique and outcome of RL repair were systematically extracted from the included studies.

\section{Results}

A total of 387 studies matched our search terms. After exclusion of duplicates, 37 studies were initially included based on their abstract. 27 studies were finally included. The average MINORS score of all non-randomised studies was 8.7/16 for non-comparative studies and 16.9/24 for comparative studies, respectively (Tables 2,3 ).

\section{Epidemiology}

The reviewed literature reports a prevalence of RLs in ACLdeficient knees ranging from 9 to $24 \%$ [3, 35]. RLs account for $17 \%-55 \%$ of all MM injuries [14, 26]. There is evidence that RLs occur more frequently in younger and paediatric patients [23, 25]. An increased medial meniscal slope [38] and prolonged time from injury to surgery $[16,23,40]$ have also been associated with a higher incidence of RLs. Sonnery-Cottet et al. [40] reported that male sex, maximum age $\leq 30$ years, revision ACL reconstruction (ACLR), side-toside laxity difference $>6 \mathrm{~mm}$, and the presence of a lateral meniscal tear are all significant risk factors for RLs. Furthermore, RLs seem to be more prevalent in contact sports injuries [35] (Table 4).

\section{Arthroscopy}

Most authors have pointed out that RLs are easily missed through a standard anterolateral arthroscopic portal [1,3, $23,39]$. Thus, exploration via a trans-notch view or an additional posteromedial portal has been commonly suggested to specifically check for these lesions. The posteromedial portal has been reported to be safe concerning possible damage of popliteal neurovascular structures $[5,9,16,30]$.

Kim et al. evaluated the accuracy of a sequential arthroscopic 4-step approach for the diagnosis of RLs [18]. The authors showed that $38 \%$ of all RLs were found using the initial standard exploration via an anterolateral portal. Fortyeight percent of RLs were identified through an intercondylar view using a $30^{\circ}$ arthroscope. A trans-notch view using a $70^{\circ}$ arthroscope and exploration through a posteromedial portal both resulted in a $100 \%$ detection rate. Additionally, the authors did not find a significant correlation between prolonged time from injury (TFI) and the overall prevalence of RLs; however, an association between TFI and the diagnostic step of detecting a RL was observed. RLs in chronic ACL tears ( $>3$ months) were more often detected through a standard anterolateral portal as compared to those in acute ACL injuries ( $<3$ months).

The difficulty of diagnosing a RL via an anterolateral portal has been confirmed by others [9, 25, 39] (Table 5).

Lesions affecting the meniscotibial ligament can be covered by an intact capsule and, therefore, remain undetected unless soft-tissue debridement is performed [9]. In case of meniscus instability without a corresponding lesion on standard arthroscopy, debridement via a posteromedial portal under trans-notch visualisation is recommended [9]. Zhang et al. [49] reported an association between a wavelike chondral injury of the medial femoral condyle and the presence of RL on arthroscopic exploration. The authors examined 1596 patients of which 4.9\% (78/1596) presented with the so-called wave sign. A RL was confirmed in all of these patients.

\section{Magnetic resonance imaging}

Numbers regarding the sensitivity of MRI for the detection of RLs are very heterogeneous, ranging from 48 to $86 \%$ [6, 41]. The specificity has been reported to range from 79 to $99 \%[2,48]$. The criteria for the diagnosis of RLs on MRI in each individual study are displayed in Table 6 . The diagnostic accuracy of hidden lesions on MRI remains unclear, since hidden lesions have not explicitly been considered in the aforementioned studies. Arthroscopy represented the gold standard for the diagnosis of RLs in all but one study; Kim et al. applied MRI as reference standard to demonstrate a correlation between the "uncovered medial meniscus sign" and an anterior tibial translation which could indicate instability of the PHMM in ACL-deficient knees [19]. The remainder of the studies applied similar methodologies, measuring the diagnostic accuracy of MRI in comparison to arthroscopy in an ACL-deficient patient collective (Table 6). 
Table 2 Patient demographics and study details extracted from studies included in the review

\begin{tabular}{|c|c|c|c|c|c|c|c|c|c|}
\hline References & Journal & Year & $\begin{array}{l}\text { No. of } \\
\text { patients }\end{array}$ & $\begin{array}{l}\text { Gender }(\% \\
\text { male) }\end{array}$ & Mean age & Study type & $\begin{array}{l}\text { Mean follow- } \\
\text { up (months) }\end{array}$ & Focus & MINORS \\
\hline $\begin{array}{l}\text { Sonnery- } \\
\text { Cottet et al. } \\
{[40]}\end{array}$ & AMJSM & 2018 & 3214 & 72.5 & NR & Case-control & 45.6 & $\begin{array}{l}\text { Epidemiology, } \\
\text { Repair (Suture- } \\
\text { Lasso) }\end{array}$ & $17 / 24$ \\
\hline $\begin{array}{l}\text { Kim et al. } \\
\text { [19] }\end{array}$ & AJR & 2018 & 85 & 83.5 & 31.6 & $\begin{array}{l}\text { Retrospective } \\
\text { analysis }\end{array}$ & $\begin{array}{l}4 \text { days } \\
\text { (trauma to } \\
\text { MRI) }\end{array}$ & Diagnosis (MRI) & $10 / 16$ \\
\hline Seil et al. [35] & KSSTA & 2018 & 224 & 64.3 & 27.0 & Case-control & NR & Epidemiology & $7 / 16$ \\
\hline $\begin{array}{l}\text { Edgar et al. } \\
\text { [10] }\end{array}$ & JAAOS & 2018 & 337 & $64.0^{\mathrm{a}}$ & 23.0 & $\begin{array}{l}\text { Cross-sec- } \\
\text { tional }\end{array}$ & NR & Epidemiology & $7 / 16$ \\
\hline $\begin{array}{c}\text { Kumar et al. } \\
{[21]}\end{array}$ & OJSM & 2018 & 852 & 55.2 & 28.6 & Case-control & NR & Epidemiology & $16 / 24$ \\
\hline $\begin{array}{l}\text { Yeo et al. } \\
\text { [48] }\end{array}$ & $\begin{array}{l}\text { Skeletal Radi- } \\
\text { ology }\end{array}$ & 2018 & 78 & 82.1 & 33.7 & $\begin{array}{l}\text { Retrospective } \\
\text { analysis }\end{array}$ & NR & Diagnosis (MRI) & $16 / 24$ \\
\hline $\begin{array}{l}\text { Hatayama } \\
\text { et al. [14] }\end{array}$ & Arthroscopy & 2018 & 155 & 51.0 & 25.3 & Case-control & $\begin{array}{l}\text { 42d (MRI to } \\
\text { ASC) }\end{array}$ & Diagnosis (MRI) & $18 / 24$ \\
\hline $\begin{array}{l}\text { Kim et al. } \\
{[18]}\end{array}$ & Arthroscopy & 2018 & 195 & 88.2 & 31.7 & Case-control & $\begin{array}{l}9.3 \text { (injury to } \\
\text { surgery) }\end{array}$ & Diagnosis (ASC) & $17 / 24$ \\
\hline $\begin{array}{l}\text { Di Vico et al. } \\
\text { [9] }\end{array}$ & MLTJ & 2017 & 115 & 90.4 & 27.0 & $\begin{array}{l}\text { Observational } \\
\text { study }\end{array}$ & NR & Diagnosis (ASC) & $11 / 16$ \\
\hline $\begin{array}{l}\text { Chen et al. } \\
\text { [5] }\end{array}$ & JNS & 2017 & 46 & 73.9 & 26.0 & $\begin{array}{l}\text { Observational } \\
\text { study }\end{array}$ & 32.0 & $\begin{array}{l}\text { Repair (FastFix, } \\
\text { Smith\&Nephew) }\end{array}$ & $10 / 16$ \\
\hline $\begin{array}{l}\text { Yang et al. } \\
\text { [47] }\end{array}$ & JMNI & 2017 & 68 & 75.0 & 35.3 & Case-control & 18.0 & $\begin{array}{l}\text { Repair (Trephina- } \\
\text { tion/Abrasion } \\
\text { vs FastFix, } \\
\text { Smith\&Nephew) }\end{array}$ & $19 / 24$ \\
\hline $\begin{array}{c}\text { DePhillipo } \\
\text { et al. [6] }\end{array}$ & AMJSM & 2017 & 301 & $66.0^{\mathrm{a}}$ & $29.6^{\mathrm{a}}$ & Case series & NR & $\begin{array}{l}\text { Epidemiology, } \\
\text { Diagnosis (MRI) }\end{array}$ & $7 / 16$ \\
\hline $\begin{array}{l}\text { Arner et al. } \\
\text { [2] }\end{array}$ & KSSTA & 2017 & 90 & 50.0 & 28.0 & Case series & $\begin{array}{l}57.3 \text { days } \\
\text { (MRI to } \\
\text { ASC) }\end{array}$ & Diagnosis (MRI) & $16 / 24$ \\
\hline $\begin{array}{l}\text { Malatray } \\
\text { et al. [25] }\end{array}$ & KSSTA & 2017 & 56 & 76.8 & 14.0 & Case series & $\begin{array}{r}11.5 \text { (trauma } \\
\text { to surgery) }\end{array}$ & Diagnosis (ASC) & $9 / 16$ \\
\hline Liu et al. [24] & AMJSM & 2017 & 91 & 74.7 & 35.2 & $\mathrm{RCT}$ & 24.0 & $\begin{array}{l}\text { Repair (Trephina- } \\
\text { tion/Abrasion } \\
\text { vs Suture Hook, } \\
\text { Linvatec) }\end{array}$ & \\
\hline $\begin{array}{l}\text { Keyhani et al. } \\
\text { [16] }\end{array}$ & KSSTA & 2017 & $128(166)$ & 83.6 & 24.0 & Case series & 24.0 & $\begin{array}{l}\text { Repair (Suture } \\
\text { Hook, Linvatec) }\end{array}$ & $13 / 16$ \\
\hline $\begin{array}{l}\text { Thaunat et al. } \\
\text { [44] }\end{array}$ & Arthroscopy & 2016 & 132 & 83.3 & 26.4 & Case series & 27.0 & $\begin{array}{l}\text { Repair (Suture } \\
\text { Lasso, Arthrex) }\end{array}$ & $12 / 16$ \\
\hline $\begin{array}{l}\text { Song et al. } \\
\text { [38] }\end{array}$ & AMJSM & 2016 & 106 & 81.1 & 26.1 & Case-control & NR & $\begin{array}{l}\text { Epidemiology } \\
\text { (increased MM } \\
\text { slope) }\end{array}$ & $16 / 24$ \\
\hline $\begin{array}{l}\text { Zhang et al. } \\
\text { [49] }\end{array}$ & $\begin{array}{l}\text { Int. Orthopae- } \\
\text { dics }\end{array}$ & 2015 & 78 & 60.3 & 26.7 & Case series & NR & $\begin{array}{l}\text { Wave sign chondral } \\
\text { injury }\end{array}$ & $6 / 16$ \\
\hline Li et al. [22] & KSRR & 2015 & 23 & NR & NR & Case series & 14.0 & $\begin{array}{l}\text { Repair (FastFix, } \\
\text { Smith\&Nephew) }\end{array}$ & $6 / 16$ \\
\hline $\begin{array}{l}\text { Sonnery- } \\
\text { Cottet et al. } \\
\text { [39] }\end{array}$ & AMJSM & 2014 & 302 & 78.8 & 28.0 & Case series & $\begin{array}{l}9.7 \text { (trauma to } \\
\text { surgery) }\end{array}$ & Diagnosis (ASC) & $8 / 16$ \\
\hline $\begin{array}{l}\text { Kijowski } \\
\text { et al. [17] }\end{array}$ & $\begin{array}{l}\text { Musculoskel- } \\
\text { etal Imaging }\end{array}$ & 2014 & 64 & 62.5 & 25.2 & Case series & $\begin{array}{l}\text { 22d (trauma } \\
\text { to MRI), } \\
\text { 48d (MRI } \\
\text { to surgery), } \\
\text { 70d (trauma } \\
\text { to surgery) }\end{array}$ & Diagnosis (MRI) & $6 / 16$ \\
\hline
\end{tabular}


Table 2 (continued)

\begin{tabular}{|c|c|c|c|c|c|c|c|c|c|}
\hline References & Journal & Year & $\begin{array}{l}\text { No. of } \\
\text { patients }\end{array}$ & $\begin{array}{l}\text { Gender }(\% \\
\text { male })\end{array}$ & Mean age & Study type & $\begin{array}{l}\text { Mean follow- } \\
\text { up (months) }\end{array}$ & Focus & MINORS \\
\hline $\begin{array}{l}\text { Furumatsu } \\
\text { et al. [11] }\end{array}$ & $\begin{array}{l}\text { Int. Orthopae- } \\
\text { dics }\end{array}$ & 2013 & 20 & 40.0 & 19.0 & Case series & 24.0 & $\begin{array}{l}\text { Repair (FastFix, } \\
\text { Smith\&Nephew) }\end{array}$ & $12 / 16$ \\
\hline Liu et al. [23] & AMJSM & 2011 & 868 & $78.5^{\mathrm{a}}$ & $24.7^{\mathrm{a}}$ & $\begin{array}{l}\text { Cross-sec- } \\
\text { tional }\end{array}$ & $\begin{array}{c}27.2 \text { (trauma } \\
\text { to surgery) }\end{array}$ & Epidemiology & $7 / 16$ \\
\hline $\begin{array}{l}\text { Bollen et al. } \\
\text { [3] }\end{array}$ & JBJS & 2010 & 183 & NR & NR & $\begin{array}{l}\text { Cross-sec- } \\
\text { tional }\end{array}$ & NR & Epidemiology & $7 / 16$ \\
\hline $\begin{array}{l}\text { Smith et al. } \\
\text { [37] }\end{array}$ & AJSM & 2001 & 575 & 63.7 & 25.4 & $\begin{array}{l}\text { Cross-sec- } \\
\text { tional }\end{array}$ & NR & Epidemiology & $12 / 16$ \\
\hline $\begin{array}{l}\text { Rubin et al. } \\
\text { [33] }\end{array}$ & $\begin{array}{l}\text { Musculoskel- } \\
\text { etal Radiol- } \\
\text { ogy }\end{array}$ & 1996 & 52 & 75.0 & 32.0 & Case series & $\begin{array}{l}\text { 46d (MRI to } \\
\text { surgery) }\end{array}$ & Diagnosis (MRI) & $6 / 16$ \\
\hline
\end{tabular}

AMJSM American Journal of Sports Medicine, AJR American Journal of Roentgenology, JAAOS Journal of the American Academy of Orthopedic Surgeons, JBJS Journal of Bone and Joint Surgery, JMNI Journal of Musculoskeletal and Neuronal Interactions, JNS The Journal of Knee Surgery, JOSR Journal of Orthopaedic Surgery and Research, KSRR Knee Surgery and Related Research, KSSTA Knee Surgery Sports Traumatology Arthroscopy, MLTJ Muscles, Ligaments and Tendons Journal, OJSM Orthopedic Journal of Sports Medicine, OTSR Orthopaedics and Traumatology: Surgery and Research

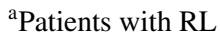

Table 3 Methodological index for non-randomised studies (MINORS)

\begin{tabular}{|c|c|c|c|c|c|c|c|c|c|c|c|c|c|}
\hline References & 1 & 2 & 3 & 4 & 5 & 6 & 7 & 8 & 9 & 10 & 11 & 12 & Total \\
\hline Sonnery-Cottet et al. [40] & 2 & 2 & 2 & 2 & 0 & 2 & 1 & 0 & 2 & 2 & 0 & 2 & $17 / 24$ \\
\hline Kim Y et al. [19] & 2 & 2 & 1 & 2 & 0 & 1 & 2 & 0 & - & - & - & - & $10 / 16$ \\
\hline Seil et al. [35] & 2 & 1 & 2 & 2 & 0 & 0 & 0 & 0 & - & - & - & - & $7 / 16$ \\
\hline Edgar et al. [10] & 2 & 2 & 1 & 2 & 0 & 0 & 0 & 0 & - & - & - & - & $7 / 16$ \\
\hline Kumar et al. [21] & 2 & 2 & 1 & 2 & 2 & 0 & 0 & 0 & 2 & 2 & 1 & 2 & $16 / 24$ \\
\hline Yeo et al. [48] & 2 & 2 & 1 & 2 & 2 & 0 & 0 & 0 & 1 & 2 & 2 & 2 & $16 / 24$ \\
\hline Hatayama et al. [14] & 2 & 2 & 2 & 2 & 1 & 1 & 2 & 1 & 1 & 2 & 0 & 2 & $18 / 24$ \\
\hline Kim et al. [18] & 2 & 2 & 2 & 2 & 0 & 0 & 0 & 2 & 1 & 2 & 0 & 2 & $17 / 24$ \\
\hline Di Vico et al. [9] & 1 & 2 & 2 & 2 & 0 & 2 & 2 & 0 & - & - & - & - & $11 / 16$ \\
\hline Chen et al. [5] & 1 & 2 & 1 & 2 & 0 & 2 & 2 & 0 & - & - & - & - & $10 / 16$ \\
\hline Yang et al. [47] & 2 & 2 & 1 & 2 & 0 & 2 & 2 & 0 & 2 & 2 & 2 & 2 & $19 / 24$ \\
\hline DePhillipo et al. [6] & 2 & 2 & 1 & 2 & 0 & 0 & 0 & 0 & - & - & - & - & $7 / 16$ \\
\hline Arner et al. [2] & 2 & 2 & 0 & 2 & 2 & 0 & 0 & 0 & 2 & 2 & 2 & 2 & $16 / 24$ \\
\hline Malatray et al. [25] & 2 & 2 & 2 & 2 & 0 & 0 & 0 & 1 & - & - & - & - & $9 / 16$ \\
\hline Keyhani et al. [16] & 2 & 2 & 2 & 2 & 0 & 2 & 1 & 2 & - & - & - & - & $13 / 16$ \\
\hline Thaunat et al. [44] & 2 & 2 & 2 & 2 & 0 & 2 & 2 & 0 & - & - & - & - & $12 / 16$ \\
\hline Song et al. [38] & 2 & 2 & 0 & 2 & 2 & 0 & 0 & 0 & 2 & 2 & 2 & 2 & $16 / 24$ \\
\hline Zhang et al. [49] & 1 & 2 & 1 & 2 & 0 & 0 & 0 & 0 & - & - & - & - & $6 / 16$ \\
\hline Li et al. [22] & 0 & 0 & 0 & 2 & 0 & 2 & 2 & 0 & - & - & - & - & $6 / 16$ \\
\hline Sonnery-Cottet et al. [39] & 2 & 2 & 2 & 2 & 0 & 0 & 0 & 0 & - & - & - & - & $8 / 16$ \\
\hline Kijowski et al. [17] & 1 & 0 & 1 & 2 & 2 & 0 & 0 & 0 & - & - & - & - & $6 / 16$ \\
\hline Furumatsu et al. [11] & 2 & 2 & 0 & 2 & 2 & 2 & 2 & 0 & - & - & - & - & $12 / 16$ \\
\hline Liu et al. [23] & 2 & 2 & 1 & 2 & 0 & 0 & 0 & 0 & - & - & - & - & $7 / 16$ \\
\hline Bollen et al. [3] & 1 & 2 & 2 & 2 & 0 & 0 & 0 & 0 & - & - & - & - & $7 / 16$ \\
\hline Smith et al. [37] & 2 & 2 & 2 & 2 & 2 & 2 & 0 & 0 & - & - & - & - & $12 / 16$ \\
\hline Rubin et al. [33] & 2 & 0 & 1 & 2 & 1 & 0 & 0 & 0 & - & - & - & - & $6 / 16$ \\
\hline
\end{tabular}

$2=$ reported and adequate, $1=$ reported, but inadequate, $0=$ not reported; max. score 16 (non-comparative studies) and 24 (comparative studies) 
Table 4 Arthroscopic prevalence of ramp lesions in ACL-deficient knees

\begin{tabular}{|c|c|c|c|c|c|c|c|}
\hline Authors & Year & $N$ & Selection criteria & Mean age & TFI (months) & Tear type & Prevalence $(\%)$ \\
\hline Sonnery-Cottet et al. [40] & 2018 & 3214 & $\begin{array}{l}\text { Primary or revision } \\
\text { ACLR }\end{array}$ & 28.0 & 4.9 & RL & 23.9 \\
\hline \multirow[t]{2}{*}{ Seil et al. [35] } & \multirow[t]{2}{*}{2018} & \multirow[t]{2}{*}{224} & \multirow[t]{2}{*}{ ACL injury } & \multirow[t]{2}{*}{27.4} & \multirow[t]{2}{*}{4.6} & MM & 41.0 \\
\hline & & & & & & RL & $55.0^{\mathrm{a}}, 24.0$ \\
\hline Edgar et al. [10] & 2018 & 337 & Primary ACLR & 26.0 & NR & Posteromedial MCS & 13.1 \\
\hline Yeo et al. [48] & 2018 & 78 & ACL injury & 33.7 & NR & RL & 9.0 \\
\hline \multirow[t]{2}{*}{ Kumar et al. [21] } & \multirow[t]{2}{*}{2018} & \multirow[t]{2}{*}{852} & \multirow[t]{2}{*}{ ACL injury } & \multirow[t]{2}{*}{29.2} & \multirow[t]{2}{*}{ NR } & MM & 36.0 \\
\hline & & & & & & RL & $41.4^{\mathrm{a}}, 14.9$ \\
\hline Kim SH et al. [18] & 2018 & 195 & $\begin{array}{l}\text { Acute or chronic ACL } \\
\text { injury }\end{array}$ & 31.7 & NR & RL & 26.6 \\
\hline \multirow[t]{2}{*}{ Mansori et al. [26] } & \multirow[t]{2}{*}{2018} & \multirow[t]{2}{*}{362} & \multirow[t]{2}{*}{ ACL injury } & \multirow[t]{2}{*}{32.1} & \multirow[t]{2}{*}{11.7} & MM & 40.6 \\
\hline & & & & & & $\begin{array}{l}\text { MM peripheral } \\
(\mathrm{MCS}+\mathrm{RL})\end{array}$ & 5.0 \\
\hline \multirow[t]{2}{*}{ Hatayama et al. [14] } & \multirow[t]{2}{*}{2018} & \multirow[t]{2}{*}{155} & \multirow[t]{2}{*}{ ACL injury } & \multirow[t]{2}{*}{25.3} & \multirow[t]{2}{*}{ NR } & MM & 52.3 \\
\hline & & & & & & RL & $29.7^{\mathrm{a}}$ \\
\hline \multirow[t]{3}{*}{ Di Vico et al. [9] } & \multirow[t]{3}{*}{2017} & \multirow[t]{3}{*}{115} & \multirow[t]{3}{*}{ ACL injury } & \multirow[t]{3}{*}{27.0} & \multirow[t]{3}{*}{10.0} & $\begin{array}{l}\text { Longitudinal lesions that } \\
\text { involve the peripheral } \\
\text { attachment of the } \\
\text { PHMM }\end{array}$ & 9.6 \\
\hline & & & & & & RL & 7.8 \\
\hline & & & & & & HL & 1.7 \\
\hline DePhillippo et al. [6] & 2017 & 301 & ACL injury $+\mathrm{MM}$ tear & NR & NR & RL & $16.6^{\mathrm{a}}$ \\
\hline Malatray et al. [25] & 2017 & 56 & ACL injury & 13.9 & 20.2 & RL & 23.2 \\
\hline Arner et al. & 2017 & 90 & ACLR & 28.0 & NR & RL & 14.4 \\
\hline Song et al. [38] & 2016 & 1012 & Non-contact ACL injury & 26.1 (RL only) & NR & RL & 15.8 \\
\hline Keyhani et al. [16] & 2016 & 927 & ACL injury & 24.0 & NR & RL & 17.9 \\
\hline Shelbourne et al. [36] & 2015 & 3385 & ACL injury & 21.5 & NR & $\begin{array}{l}\text { Vertical tears in the } \\
\text { periphery of the PHMM } \\
\text { at least } 1 \mathrm{~cm} \text { in length }\end{array}$ & 12.4 \\
\hline Peltier et al. [30] & 2015 & 39 & ACL injury & 33.0 & NR & Presumably RL + HL & 12.8 \\
\hline \multirow[t]{3}{*}{ Sonnery-Cottet et al. [39] } & 2014 & 302 & ACL injury & NR & NR & MM & 41.4 \\
\hline & & & & & & RL & 9.6 \\
\hline & & & & & & HL & 7.0 \\
\hline Liu et al. [23] & 2011 & 868 & ACL injury & NR & NR & RL & 16.6 \\
\hline Bollen et al. [3] & 2010 & 183 & ACL injury & NR & NR & posteromedial MCS & 9.3 \\
\hline Smith et al. [37] & 2001 & 575 & $\begin{array}{l}\text { ACL injury + any menis- } \\
\text { cus tear }\end{array}$ & 25.4 & NR & $\begin{array}{l}\text { Peripheral posterior horn } \\
\text { tears of the medial } \\
\text { meniscus }\end{array}$ & $40^{\mathrm{a}}$ \\
\hline
\end{tabular}

TFI time from injury (to surgery); mean age and TFI are point estimates; percentages of prevalence refer to all included patients unless stated otherwise

${ }^{\mathrm{a}} \mathrm{MM}$ tears

\section{Outcome}

Eight studies were identified which report on the outcome of RL repair in a total of 855 ACL-deficient knees. The average age of patients at the time of surgery was 28.4 years and $74.9 \%$ (623/832) were males. Among the eight studies, one randomised controlled trial (RCT) [24], and two retrospective case-control studies were identified [40, 47] (Table 7).
For RL repair, all studies used an all-inside technique. A variety of fixation systems (Smith \& Nephew FasT-Fix [5, 22, 47], DePuy Synthes Omnispan [45], ConMed-Linvatec Suture-Hook and Arthrex Suture-Lasso [40, 44]) was used.

A range of different parameters regarding the outcome of RL repair in knees with concomitant ACL injuries has been reported in the literature. Among these are the Lysholm Knee Score (LNS), International Knee Documentary Committee (IKDC), Barrett criteria (presence of joint tenderness, 
effusion, and McMurray test) and healing status as per second-look arthroscopy or MRI. The LNS and IKDC Score were found to be the most frequently reported outcome parameters.

RL repair leads to a significant improvement of subjective knee scores, regardless of the specific technique (Table 7).

In a recent study by Sonnery-Cottet et al. [40] RL repair was performed in 769 patients suffering from acute or chronic ACL injury, using the Arthrex Suture Lasso. The patients were divided into the following two groups: ACLR only + RL repair; and ACLR + anterolateral ligament repair $(A L L R)+R L$ repair. Failure of RL repair was defined as the performance of secondary meniscectomy within the followup period of an average 45.6 months. The authors reported that patients who underwent ACLR + ALLR had a > twofold lower risk for reoperation due to failure of RL repair as compared to patients who underwent ACLR $+R L$ repair. This study demonstrates that ALLR can have a protective effect on RL repair; yet, the effect of RL repair itself was not analysed.

Liu et al. conducted a randomised controlled trial, including 73 patients with ACL injury and a concomitant stable RL [24]. Criteria for stable RLs included a lack of excessive anterior translation of the PHMM on probing from the anteromedial portal and a maximum lesion length of $1.5 \mathrm{~cm}$ measured from the posteromedial portal. Patients underwent all-inside surgical repair (Suture hook, Linvatec) or abrasion and trephination only. Patients were followed up for at least 24 months evaluating both subjective and objective parameters. In terms of functional scores (LNS and IKDC), there was no significant difference between the two groups. Additionally, no difference in knee stability (pivot-shift test, Lachmann test, KT-1000 arthrometer) was observed. Finally, healing status was assessed on T2-weighted sagittal MRI scans, showing that there was no statistically significant difference between the study group and control group. In conclusion, this study indicates that all-inside repair of stable $\mathrm{RL}$ is non-superior to abrasion and trephination alone.

In a retrospective controlled analysis, Yang et al. compared arthroscopic abrasion and trephination and Fast-Fix repair of RLs measuring 1-2 cm with concomitant ACL injury in 68 patients [47]. At a minimum of 24 months after surgery, there was no significant difference between the groups, indicating that arthroscopic refreshment of stable RLs achieves similar results compared to Fast-Fix repair (Table 7).

\section{Discussion}

The most important finding of this study was that ramp lesions are frequently missed in ACL-deficient knees and should be repaired in case of instability. 


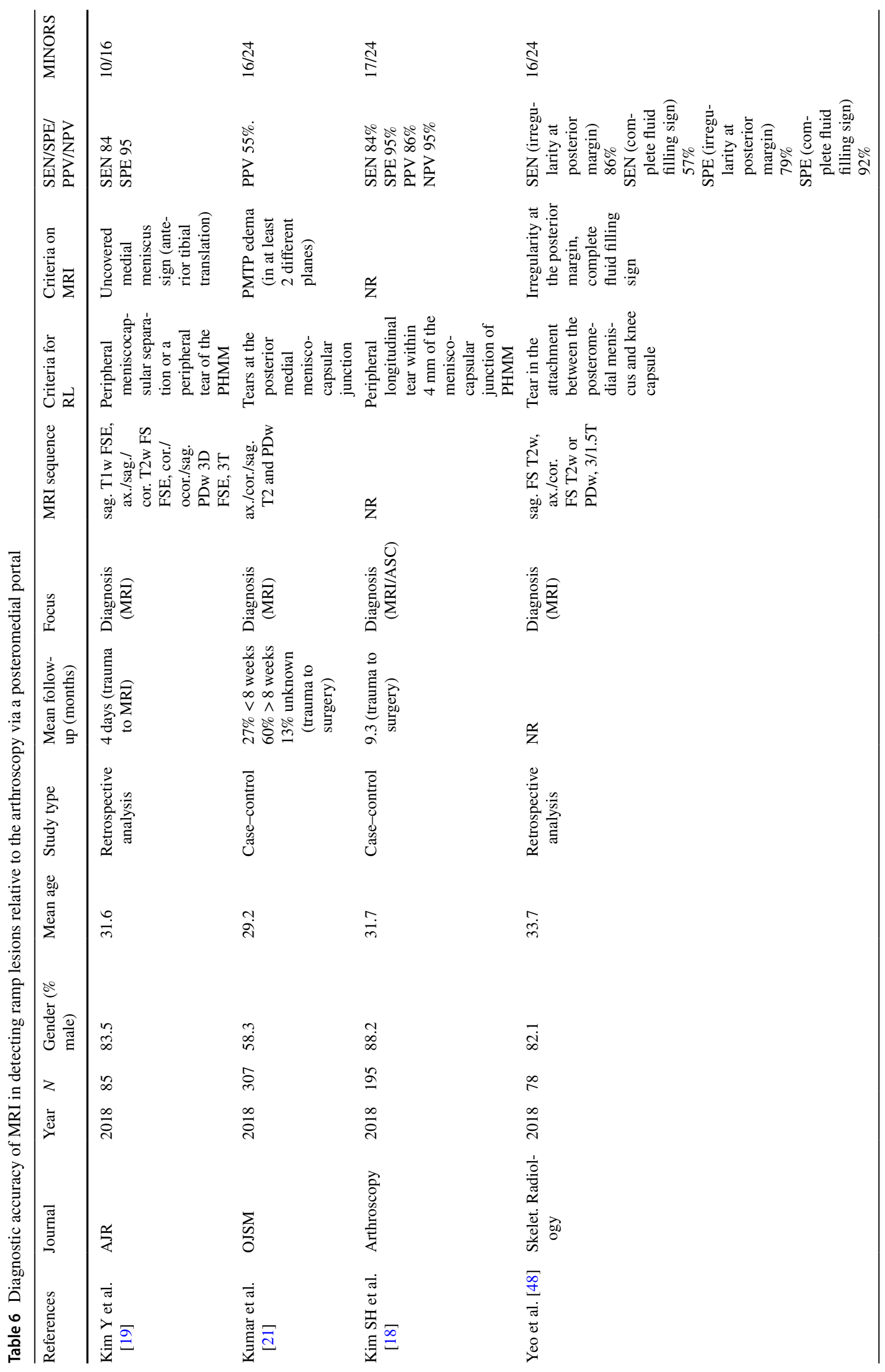




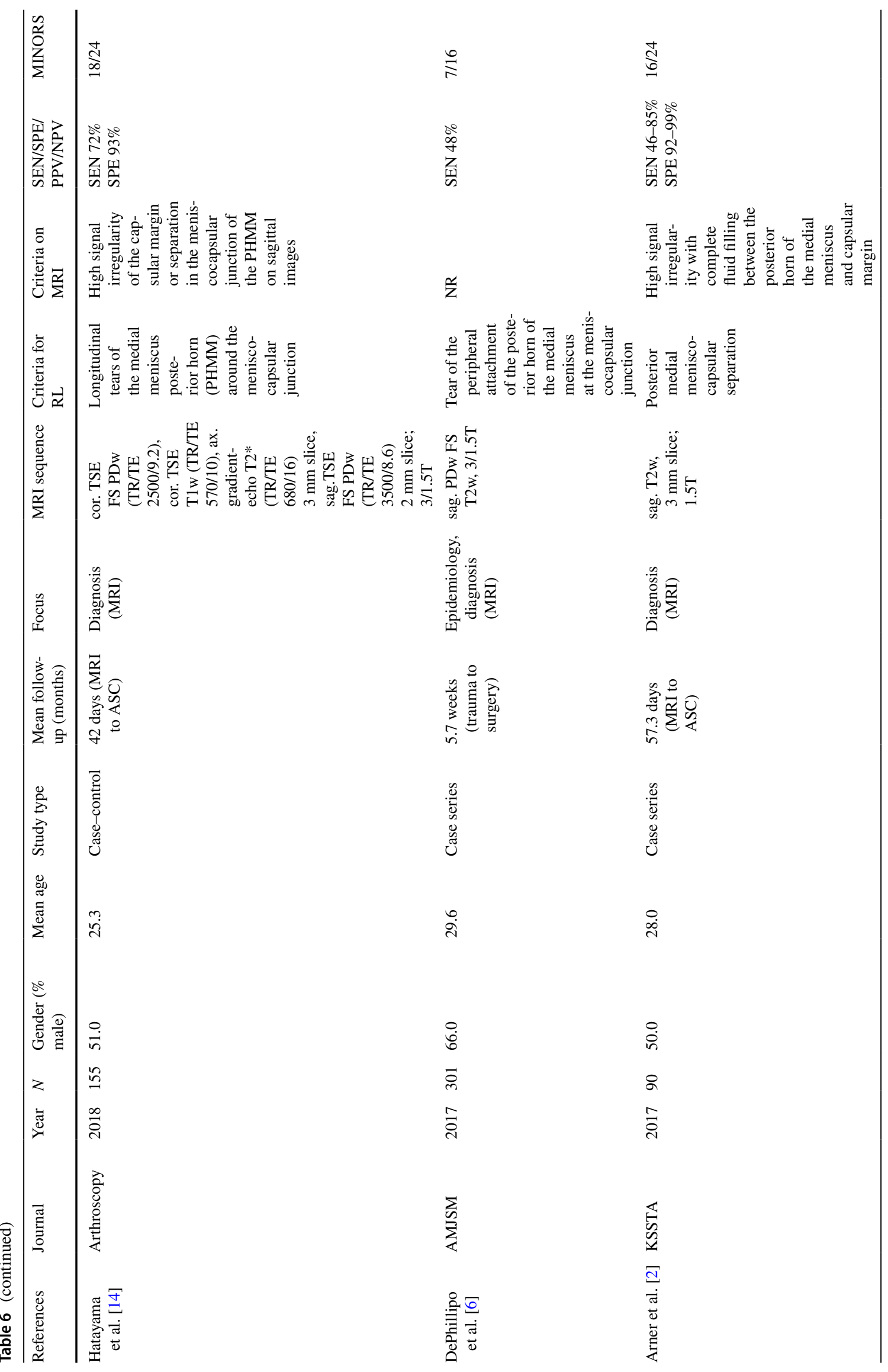




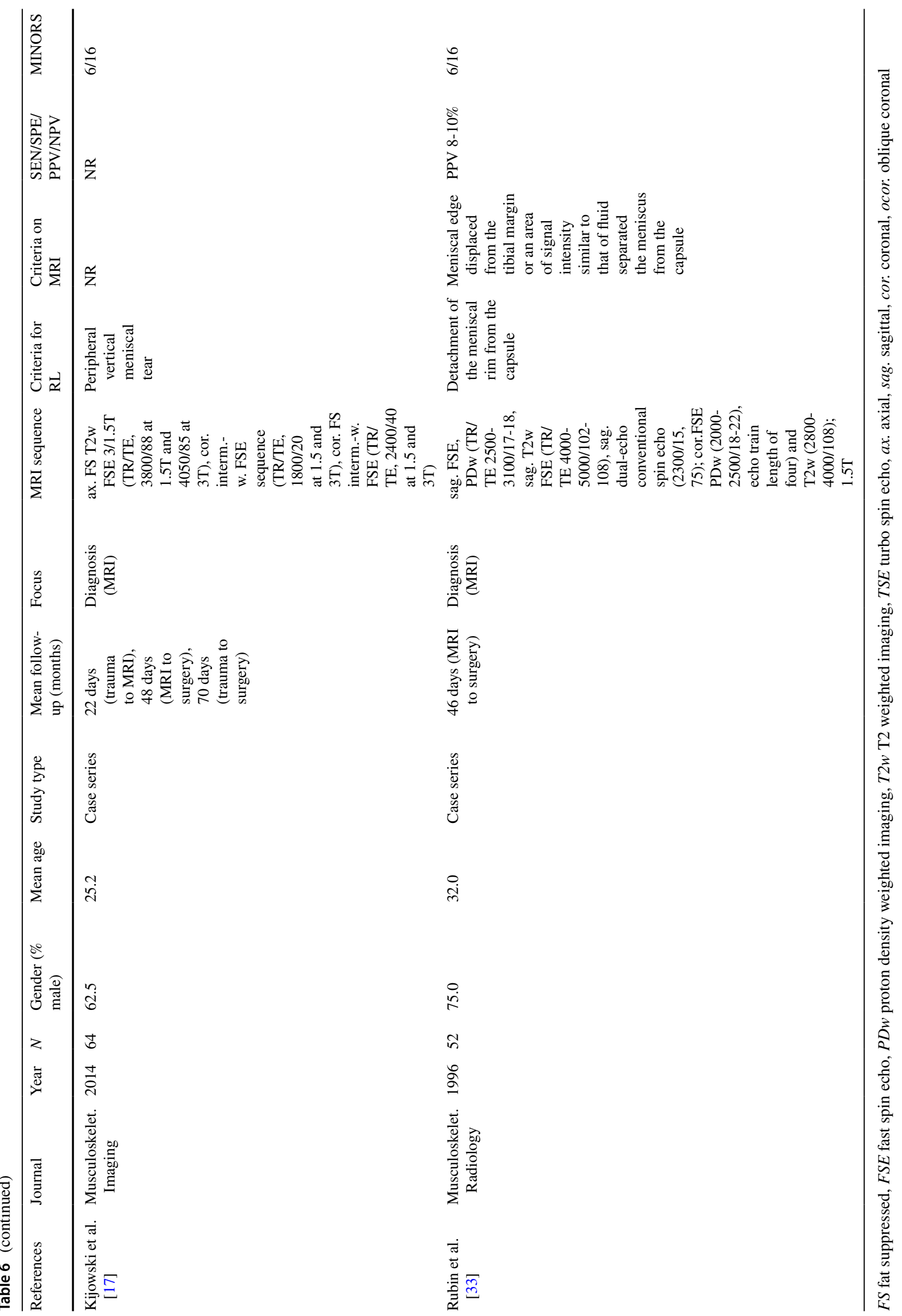


Table 7 Overview of the clinical outcome following ramp lesion repair

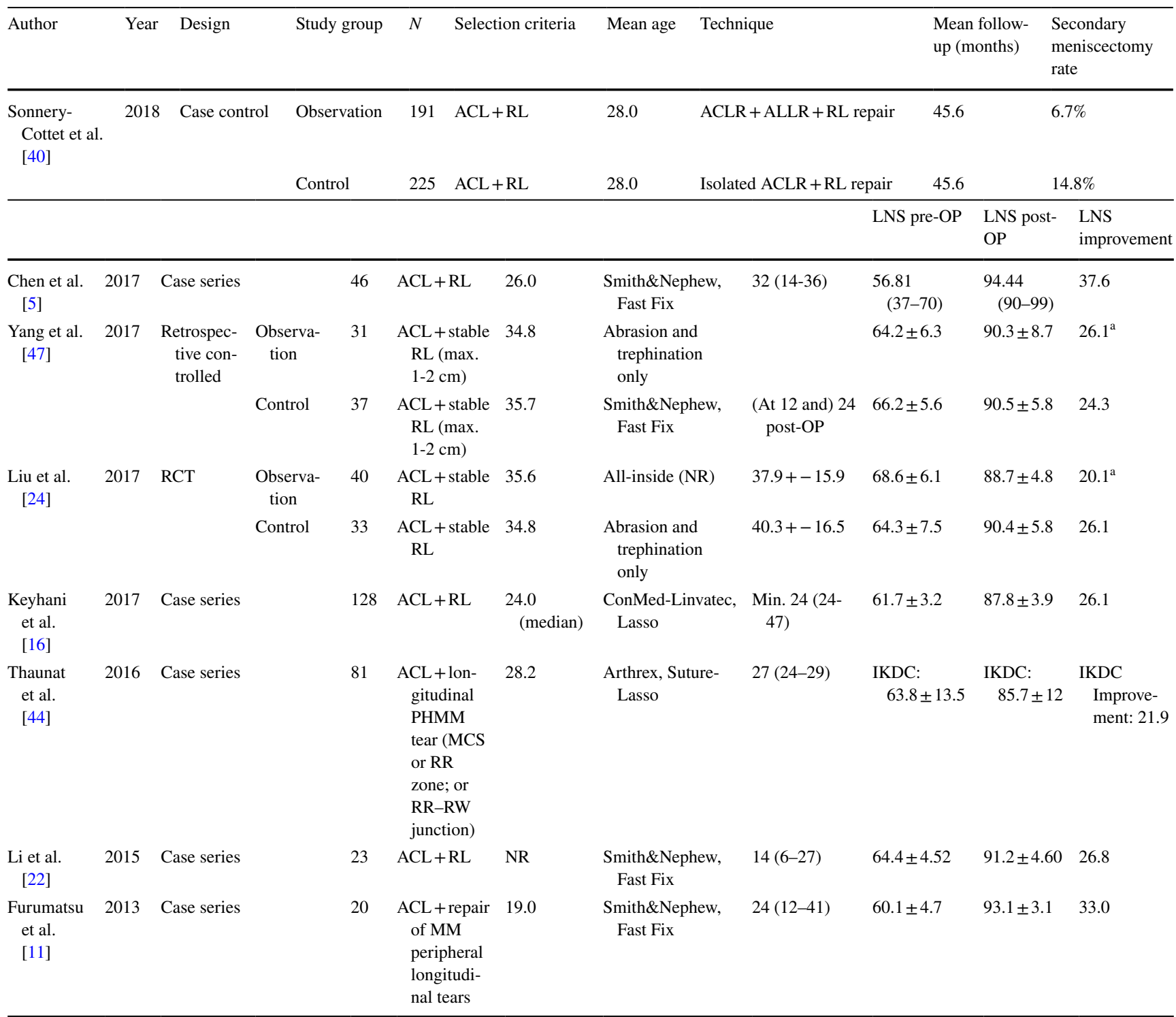

$R C T$ randomised controlled trial, LNS Lysholm Knee Score, IKDC International Knee Documentation Committee

${ }^{a}$ No significant difference as compared to control group

Considering the high prevalence of RLs in ACL-deficient knees, exploration of the posteromedial meniscocapsular complex through a posteromedial (PM) portal seems indicated if a lesion is suspected $[1,15,24,40]$. Recently, Kim et al. demonstrated that a trans-notch view using a $70^{\circ}$ arthroscope can achieve similar results in terms of RL detection rates [18]. However, an additional posteromedial portal should be considered the gold standard for the diagnosis of ramp lesions. Furthermore, a PM portal offers the benefit of a full exploration of the lesion extent and allows for a dynamic assessment of instability in flexion. If meniscus instability is observed at probing and no obvious lesion can be identified, soft-tissue debridement via a posteromedial portal under trans-notch visualisation could aid the identification of hidden lesions [9].

The current literature depicts limited and quite heterogeneous data on the diagnostic accuracy of MRI to detect RLs. Arner et al. demonstrated that MRI can be useful to rule out RLs [2]. MRI has a lower sensitivity and accuracy for RLs than for meniscus body tears [14]. On MRI, fluid filling between the posterior horn of the medial meniscus and the capsular margin can be indicative of a RL [14, 48].

Based on the available case series, RL repair leads to a significant improvement of subjective knee scores, 
regardless of the specific technique. With LNS improvements between 20.1 [24] and 37.6 [5], most authors report significantly higher scores postoperatively as compared to preoperatively. These findings are in accordance with the results of a few case series reporting excellent healing results from 87 to $95 \%$ as evaluated during second-look arthroscopy [5, 24]. Biomechanical studies have also demonstrated that RL repair can restore knee stability when simultaneous ACLR is performed [7, 41]. A recent study by SonneryCottet et al. demonstrated a significantly decreased risk of RL repair failure in patients undergoing concomitant ACLR and anterolateral ligament reconstruction, as compared to concomitant ACLR only [40]. For stable RLs, repair tends to be non-superior to trephination and abrasion only [24, 47]. This has also been shown by the only available randomised controlled study by Liu et al. [24].

There is a considerable inconsistency regarding the nomenclature of ramp lesions in the literature [34, 43]. The term ramp lesion has frequently been used as an umbrella term indicating posteromedial meniscus tears, rather than a specific type of tear (Table 1). Both tears of the meniscocapsular and meniscotibial attachment sites of the PHMM, as well as tears in the RR zone, have been termed ramp lesions $[5,9,23,43]$. To allow a comparison of studies, the authors of this work suggest to only consider meniscocapsular and meniscotibial lesions as ramp lesion. This is supported by a recent experimental study of DePhilippo et al. reporting that the meniscocapsular and meniscotibial attachments merge at a common attachment at the PHMM [8].

The following limitations have to be acknowledged: (1) generalised recommendations for the treatment of ramp lesions cannot be made based on the available literature. Ramp lesion repair leads to significant improvements in the clinical outcome; however, only two studies compared different surgical treatment options (trephination and abrasion vs RL repair). (2) Different ACLR techniques might lead to a different outcome. This review did not account for different fixation techniques. (3) Some authors considered lesions of the meniscocapsular attachment site and lesions at the periphery of the red-red zone as ramp lesions. Therefore, in a limited number of cases (Table 1), the reported postoperative improvements of RL repair may not exclusively be related to meniscocapsular lesions. (4) None of the MRI studies considered hidden lesions explicitly; therefore, it remains unclear whether these lesions can preoperatively be detected on MRI.

This work reports on the high prevalence of peripheral posteromedial meniscus lesions in ACL-deficient knees and provides an overview of common ramp lesion classification systems. A better understanding of the preoperative and intraoperative diagnostic accuracy of ramp lesions is of high clinical relevance. In daily practice, a thorough investigation of the posteromedial meniscus should always be performed during ACL reconstruction. Repair is indicated in case of instability.

\section{Conclusion}

Ramp lesions are common in ACL-deficient knees and are often missed through standard anterior portals. Exploration of the meniscocapsular complex via an additional posteromedial portal is recommended, if MRI suggests a lesion in this area or if instability is present at probing. If the posteromedial view still does not reveal any lesion despite significant instability, soft-tissue debridement might uncover hidden lesions (meniscotibial ligament disruptions).

Generalised recommendations for the treatment of ramp lesions cannot be made based on the available literature. Ramp lesion repair leads to a significant improvement of subjective knee scores, regardless of the specific fixation technique. For stable ramp lesions, trephination and abrasion might be equivalent to ramp lesion repair in terms of postoperative stability. In case of instability at probing, repair should be performed.

Acknowledgements Open access funding provided by Medical University of Vienna.

Author contributions $\mathrm{AB}$ performed the systematic review and drafted the manuscript. UK supported the systematic review process and drafted the manuscript. MH revised the manuscript and supported the review process. TT provided suggestions on the review process and wrote substantial parts of the manuscript. SH analysed the data and contributed to the manuscript. RW analysed the data and contributed to the manuscript. WW directly supervised the systematic review and finalised the manuscript. All authors read and approved the final manuscript.

Funding No funding has been received for this study.

\section{Compliance with ethical standards}

Conflict of interest The authors declare that they have no competing interests.

Ethical approval This article does not contain any studies with human participants performed by any of the authors.

Informed consent For this tye of study formal consent is not required.

Open Access This article is distributed under the terms of the Creative Commons Attribution 4.0 International License (http://creativeco mmons.org/licenses/by/4.0/), which permits unrestricted use, distribution, and reproduction in any medium, provided you give appropriate credit to the original author(s) and the source, provide a link to the Creative Commons license, and indicate if changes were made. 


\section{Appendix}

\section{Database query strings}

\section{PubMed}

(tibial meniscus injuries[MeSH Terms] AND ("ramp" OR "hidden" OR "meniscocapsular" OR "meniscosynovial” OR "posteromedial")) OR

("ramp"[Tiab] AND lesion[Tiab] AND (meniscus[Tiab]

OR meniscal[Tiab])) OR

(hidden[Tiab] AND lesion[Tiab] AND (meniscus[Tiab]

OR meniscal[Tiab])) OR

(meniscocapsular[Tiab] AND (lesion[Tiab] OR “tears"[Tiab] OR “tear”[Tiab] OR “separation"[Tiab])) OR (meniscosynovial[Tiab] AND (lesion[Tiab] OR “tears"[Tiab] OR “tear"[Tiab] OR “separation"[Tiab])) OR (peripheral [Tiab] AND meniscus[Tiab] AND ("medial"[Tiab] OR "posteromedial"[Tiab]) AND (“tears"[Tiab] OR “tear"[Tiab] OR “separation”[Tiab])) AND (English[lang] OR German[lang])

\section{Scopus}

TITLE-ABS-KEY ( ( ramp AND lesion ) OR ( meniscocapsular AND ( separation OR tear OR lesion OR injury ) ) OR ( meniscosynovial AND ( separation OR tear OR lesion OR injury ) ) OR ( hidden AND lesion ) OR meniscotibial AND ligament AND ( separation OR tear OR lesion OR injury ) )

\section{References}

1. Ahn JH, Bae TS, Kang K-S, Kang SY, Lee SH (2011) Longitudinal tear of the medial meniscus posterior horn in the anterior cruciate ligament-deficient knee significantly influences anterior stability. Am J Sports Med 39:2187-2193

2. Arner JW, Herbst E, Burnham JM, Soni A, Naendrup J-H, Popchak A, Fu FH, Musahl V (2017) MRI can accurately detect meniscal ramp lesions of the knee. Knee Surg Sports Traumatol Arthrosc 25:3955-3960

3. Bollen SR (2010) Posteromedial meniscocapsular injury associated with rupture of the anterior cruciate ligament: a previously unrecognised association. J Bone Joint Surg Br 92:222-223

4. Chahla J, Dean CS, Moatshe G, Mitchell JJ, Cram TR, Yacuzzi C, LaPrade RF (2016) Meniscal ramp lesions: anatomy, incidence, diagnosis, and treatment. Orthop J Sports Med 4:2325967116657815

5. Chen Z, Li W-P, Yang R, Song B, Jiang C, Hou J-Y, Luo H, Zhou Y-F (2018) Meniscal ramp lesion repair using the FasT-Fix technique: evaluating healing and patient outcomes with second-look arthroscopy. J Knee Surg 31:710-715

6. DePhillipo NN, Cinque ME, Chahla J, Geeslin AG, Engebretsen L, LaPrade RF (2017) Incidence and detection of meniscal ramp lesions on magnetic resonance imaging in patients with anterior cruciate ligament reconstruction. Am J Sports Med 45:2233-2237
7. DePhillipo NN, Moatshe G, Brady A, Chahla J, Aman ZS, Dornan GJ, Nakama GY, Engebretsen L, LaPrade RF (2018) Effect of meniscocapsular and meniscotibial lesions in ACL-deficient and ACL-reconstructed knees: a biomechanical study. Am J Sports Med 46:2422-2431

8. DePhillipo NN, Moatshe G, Chahla J, Aman ZS, Storaci HW, Morris ER, Robbins CM, Engebretsen L, LaPrade RF (2019) Quantitative and qualitative assessment of the posterior medial meniscus anatomy: defining meniscal ramp lesions. Am J Sports Med 47:372-378

9. Di Vico G, Di Donato SL, Balato G, Correra G, D’Addona A, Maffulli N, Rosa D (2017) Correlation between time from injury to surgery and the prevalence of ramp and hidden lesions during anterior cruciate ligament reconstruction. A new diagnostic algorithm. Muscles Ligaments Tendons J 7:491-497

10. Edgar C, Kumar N, Ware JK, Ziegler C, Reed DN, DiVenere J, Obopilwe E, Cote MP, Arciero RA (2019) Incidence of posteromedial meniscocapsular separation and the biomechanical implications on the anterior cruciate ligament. J Am Acad Orthop Surg 27:e184-e192

11. Furumatsu T, Miyazawa S, Tanaka T, Okada Y, Fujii M, Ozaki $\mathrm{T}$ (2014) Postoperative change in medial meniscal length in concurrent all-inside meniscus repair with anterior cruciate ligament reconstruction. Int Orthop 38:1393-1399

12. Hagino $T$, Ochiai $S$, Senga $S$, Yamashita $T$, Wako M, Ando $T$, Haro H (2015) Meniscal tears associated with anterior cruciate ligament injury. Arch Orthop Trauma Surg 135:1701-1706

13. Hamberg P, Gillquist J, Lysholm J (1983) Suture of new and old peripheral meniscus tears. J Bone Joint Surg Am 65:193-197

14. Hatayama K, Terauchi M, Saito K, Aoki J, Nonaka S, Higuchi H (2018) Magnetic resonance imaging diagnosis of medial meniscal ramp lesions in patients with anterior cruciate ligament injuries. Arthroscopy 34:1631-1637

15. Jan N, Sonnery-Cottet B, Fayard J-M, Kajetanek C, Thaunat M (2016) Complications in posteromedial arthroscopic suture of the medial meniscus. Orthop Traumatol Surg Res 102:S287-S293

16. Keyhani S, Ahn JH, Verdonk R, Soleymanha M, Abbasian M (2017) Arthroscopic all-inside ramp lesion repair using the posterolateral transseptal portal view. Knee Surg Sports Traumatol Arthrosc 25:454-458

17. Kijowski R, Rosas HG, Lee KS, Lee KL, Cheung A, Munoz del Rio A, Graf BK (2014) MRI characteristics of healed and unhealed peripheral vertical meniscal tears. AJR Am J Roentgenol 202:585-592

18. Kim SH, Lee SH, Kim K-I, Yang JW (2018) Diagnostic accuracy of sequential arthroscopic approach for ramp lesions of the posterior horn of the medial meniscus in anterior cruciate ligamentdeficient knee. Arthroscopy 34:1582-1589

19. Kim Y, Ahn JM, Kang Y, Lee E, Lee JW, Kang HS (2018) Uncovered medial meniscus sign on knee MRI: evidence of lost brake stop mechanism of the posterior horn medial meniscus. AJR Am J Roentgenol 211:1313-1318

20. Kobayashi H, Kanamura T, Koshida S, Miyashita K, Okado T, Shimizu T, Yokoe K (2010) Mechanisms of the anterior cruciate ligament injury in sports activities: a 20-year clinical research of 1700 athletes. J Sports Sci Med 9:669-675

21. Kumar NS, Spencer T, Cote MP, Arciero RA, Edgar C (2018) Is edema at the posterior medial tibial plateau indicative of a ramp lesion? An examination of 307 patients with anterior cruciate ligament reconstruction and medial meniscal tears. Orthop J Sports Med 6:2325967118780089

22. Li W-P, Chen Z, Song B, Yang R, Tan W (2015) The FasT-Fix repair technique for ramp lesion of the medial meniscus. Knee Surg Relat Res 27:56-60 
23. Liu X, Feng H, Zhang H, Hong L, Wang XS, Zhang J (2011) Arthroscopic prevalence of ramp lesion in 868 patients with anterior cruciate ligament injury. Am J Sports Med 39:832-837

24. Liu X, Zhang H, Feng H, Hong L, Wang X-S, Song G-Y (2017) Is it necessary to repair stable ramp lesions of the medial meniscus during anterior cruciate ligament reconstruction? A prospective randomized controlled trial. Am J Sports Med 45:1004-1011

25. Malatray M, Raux S, Peltier A, Pfirrmann C, Seil R, Chotel F (2018) Ramp lesions in ACL deficient knees in children and adolescent population: a high prevalence confirmed in intercondylar and posteromedial exploration. Knee Surg Sports Traumatol Arthrosc 26:1074-1079

26. Mansori AE, Lording T, Schneider A, Dumas R, Servien E, Lustig $S$ (2018) Incidence and patterns of meniscal tears accompanying the anterior cruciate ligament injury: possible local and generalized risk factors. Int Orthop 42:2113-2121

27. Melton JTK, Murray JR, Karim A, Pandit H, Wandless F, Thomas NP (2011) Meniscal repair in anterior cruciate ligament reconstruction: a long-term outcome study. Knee Surg Sports Traumatol Arthrosc 19:1729-1734

28. Moher D, Shamseer L, Clarke M, Ghersi D, Liberati A, Petticrew M, Shekelle P, Stewart LA, PRISMA-P Group (2015) Preferred reporting items for systematic review and meta-analysis protocols (PRISMA-P) 2015 statement. Syst Rev 4:1

29. Newman JT, Carry PM, Terhune EB, Spruiell MD, Heare A, Mayo M, Vidal AF (2015) Factors predictive of concomitant injuries among children and adolescents undergoing anterior cruciate ligament surgery. Am J Sports Med 43:282-288

30. Peltier A, Lording T, Maubisson L, Ballis R, Neyret P, Lustig S (2015) The role of the meniscotibial ligament in posteromedial rotational knee stability. Knee Surg Sports Traumatol Arthrosc 23:2967-2973

31. Resnick D, Kang HS, Pretterklieber ML (2007) Internal derangements of joints, vol 2. Saunders/Elsevier, Philadelphia, pp $1631-1651$

32. Robb C, Kempshall P, Getgood A, Standell H, Sprowson A, Thompson P, Spalding T (2015) Meniscal integrity predicts laxity of anterior cruciate ligament reconstruction. Knee Surg Sports Traumatol Arthrosc 23:3683-3690

33. Rubin DA, Britton CA, Towers JD, Harner CD (1996) Are MR imaging signs of meniscocapsular separation valid? Radiology 201:829-836

34. Seil R, Hoffmann A, Scheffler S, Theisen D, Mouton C, Pape D (2017) Ramp lesions: tips and tricks in diagnostics and therapy. Orthopade 46:846-854

35. Seil R, Mouton C, Coquay J, Hoffmann A, Nührenbörger C, Pape D, Theisen D (2018) Ramp lesions associated with ACL injuries are more likely to be present in contact injuries and complete ACL tears. Knee Surg Sports Traumatol Arthrosc 26:1080-1085

36. Shelbourne KD, Benner RW, Nixon RA, Gray T (2015) Evaluation of peripheral vertical nondegenerative medial meniscus tears treated with trephination alone at the time of anterior cruciate ligament reconstruction. Arthroscopy 31:2411-2416

37. Smith JP, Barrett GR (2001) Medial and lateral meniscal tear patterns in anterior cruciate ligament-deficient knees. A prospective analysis of 575 tears. Am J Sports Med 29:415-419
38. Song G-Y, Liu X, Zhang H, Wang Q-Q, Zhang J, Li Y, Feng H (2016) Increased medial meniscal slope is associated with greater risk of ramp lesion in noncontact anterior cruciate ligament injury. Am J Sports Med 44:2039-2046

39. Sonnery-Cottet B, Conteduca J, Thaunat M, Gunepin FX, Seil R (2014) Hidden lesions of the posterior horn of the medial meniscus: a systematic arthroscopic exploration of the concealed portion of the knee. Am J Sports Med 42:921-926

40. Sonnery-Cottet B, Praz C, Rosenstiel N, Blakeney WG, Ouanezar H, Kandhari V, Vieira TD, Saithna A (2018) Epidemiological evaluation of meniscal ramp lesions in 3214 anterior cruciate ligament-injured knees from the santi study group database: a risk factor analysis and study of secondary meniscectomy rates following 769 ramp repairs. Am J Sports Med 46:3189-3197

41. Stephen JM, Halewood C, Kittl C, Bollen SR, Williams A, Amis AA (2016) Posteromedial meniscocapsular lesions increase tibiofemoral joint laxity with anterior cruciate ligament deficiency, and their repair reduces laxity. Am J Sports Med 44:400-408

42. Strobel MJ (1988) Manual of arthroscopic surgery. Springer, New York

43. Thaunat M, Fayard JM, Guimaraes TM, Jan N, Murphy CG, Sonnery-Cottet B (2016) Classification and surgical repair of ramp lesions of the medial meniscus. Arthrosc Tech 5:e871-e875

44. Thaunat M, Jan N, Fayard JM, Kajetanek C, Murphy CG, Pupim B, Gardon R, Sonnery-Cottet B (2016) Repair of meniscal ramp lesions through a posteromedial portal during anterior cruciate ligament reconstruction: outcome study with a minimum 2-year follow-up. Arthroscopy 32:2269-2277

45. Tiftikçi U, Serbest S (2017) Does the location of placement of meniscal sutures have a clinical effect in the all-inside repair of meniscocapsular tears? J Orthop Surg Res 12:87

46. Wyatt RWB, Inacio MCS, Bellevue KD, Schepps AL, Maletis GB (2017) Isolated ACL versus multiple knee ligament injury: associations with patient characteristics, cartilage status, and meniscal tears identified during ACL reconstruction. Phys Sportsmed 45:323-328

47. Yang J, Guan K, Wang JZ (2017) Clinical study on the arthroscopic refreshing treatment of anterior cruciate ligament injury combined with stable medial meniscus ramp injury. J Musculoskelet Neuronal Interact 17:108-113

48. Yeo Y, Ahn JM, Kim H, Kang Y, Lee E, Lee JW, Kang HS (2018) MR evaluation of the meniscal ramp lesion in patients with anterior cruciate ligament tear. Skeletal Radiol 47:1683-1689

49. Zhang X, You T, Jiang X, Zhang H, Zhang W (2016) Characteristic arthroscopic signs of cartilage injuries indicating concomitant occult medial meniscal peripheral tears of posterior horn. Int Orthop 40:191-195

50. Zoller SD, Toy KA, Wang P, Ebramzadeh E, Bowen RE (2017) Temporal relation of meniscal tear incidence, severity, and outcome scores in adolescents undergoing anterior cruciate ligament reconstruction. Knee Surg Sports Traumatol Arthrosc 25:215-221

Publisher's Note Springer Nature remains neutral with regard to jurisdictional claims in published maps and institutional affiliations. 